\title{
Estimation of a Permeability Field within the Two-Phase Porous Media Flow Using Nonlinear Multigrid Method
}

\author{
Tao Liu' ${ }^{1}$ and Jie Song ${ }^{2}$ \\ ${ }^{1}$ School of Mathematics and Statistics, Northeast University at Qinhuangdao, Qinhuangdao 066000, China \\ ${ }^{2}$ Department of Obstetrics and Gynecology, The Second Hospital of Jilin University, Changchun 130000, China \\ Correspondence should be addressed to Jie Song; chenjing_fuchanke@163.com
}

Received 29 October 2016; Revised 30 January 2017; Accepted 7 February 2017; Published 23 February 2017

Academic Editor: Yann Favennec

Copyright (c) 2017 Tao Liu and Jie Song. This is an open access article distributed under the Creative Commons Attribution License, which permits unrestricted use, distribution, and reproduction in any medium, provided the original work is properly cited.

\begin{abstract}
Estimation of spatially varying permeability within the two-phase porous media flow plays an important role in reservoir simulation. Usually, one needs to estimate a large number of permeability values from a limited number of observations, so the computational cost is very high even for a single field-model. This paper applies a nonlinear multigrid method to estimate the permeability field within the two-phase porous media flow. Numerical examples are provided to illustrate the feasibility and effectiveness of the proposed estimation method. In comparison with other existing methods, the most outstanding advantage of this method is the computational efficiency, computational accuracy, and antinoise ability. The proposed method has a potential applicability to a variety of parameter estimation problems.
\end{abstract}

\section{Introduction}

This paper investigates the estimation of a permeability field $q$ for the following diffusion equation within the two-phase porous media flow:

$$
\begin{aligned}
u_{t}-\nabla \cdot(q(x) N(u, \nabla u) \nabla u)=f(x, t), & \\
& (x, t) \in \Omega \times(0, T),
\end{aligned}
$$

with the initial-boundary condition

$$
\begin{aligned}
& u(x, 0)=i(x), \quad x \in \Omega, \\
& u(x, t)=b(x, t), \quad(x, t) \in \partial \Omega \times(0, T),
\end{aligned}
$$

Equation (1) originates from the partial differential equation system, which can model two-phase immiscible flow of incompressible fluids in a porous medium with zero gravity effects

$$
\begin{aligned}
& \varphi(x) S_{t}-\nabla \cdot\left(Q(x) v_{w}^{-1}(p) q_{w}(S) \nabla p\right)=f_{w}(x, t), \\
& -\varphi(x) S_{t}-\nabla \cdot\left(Q(x) v_{n}^{-1}(p) q_{n}(S)\left(\nabla p+P^{\prime}(S) \nabla S\right)\right) \\
& \quad=f_{n}(x, t),
\end{aligned}
$$

where $\varphi$ is the porosity, $S$ is the wetting-phase fluid saturation, $v$ is the fluid viscosity, $f$ is the fluid source term, $P$ is the capillary pressure, $p$ is the wetting-phase fluid pressure, $Q$ and $q$ are the absolute and relative permeabilities, and subscripts mean $w$-wetting fluid phase and $n$-nonwetting fluid phase.

$N(u, \nabla u)$ in (1) is a nonlinear function, which can model the main characteristics of the nonlinearity connected with some of the coefficient functions in (3). The nonlinearities in these two coupled nonlinear equations for $S$ and $p$ are related to both of the dependent variables. The influence of $S$ is through $S$, and the influence of $p$ is through $\nabla p$, so both $u$ and $\nabla u$ are considered as the independent variables in $N$.

Determination of the permeability coefficient $q$ of the diffusion term has shown significant potential in reservoir simulation. It can help make important decisions about the management of petroleum reservoirs, for example, recovery method, well location, fluid production, and injection rates. However, the permeability is generally modelled as a piecewise constant function; namely, it is defined by a single value within each reservoir simulator grid cell. Thus one is interested in inferring as many parameters as there are grid cells in the simulator. This number is frequently more 
than $10^{5}$ for a single field-model. There are principally two difficulties about this.

Firstly, there is no enough sufficient information in measurable reservoir quantities (well pressures and flow rates) to estimate the permeability field with such a high resolution. The usual solutions are (1) to reduce the degrees of freedom in parameter space, or (2) to use prior knowledge about the permeability to aid in the estimation, or both. Such issues are not the theme of this paper; the interested reader is directed to referenced texts [1-4] for solution.

Secondly, the computational complexity of this problem is very large, even though the number of parameters is modest corresponding to the attainable resolution implicitly given by the measurements. It may take several hours to complete reservoir simulation for a single field-model, and it needs many such simulator runs for the estimation of a permeability field. In addition, the attainable permeability resolution is expected to increase in the near future, because of the appearance of the novel data acquisition techniques, such as time-lapse $3 \mathrm{D}$ seismic surveys. Therefore, there will be an urgent need for parameter estimation methodology able to handle a larger number of parameters within a reasonable time span. The aim of this paper is to develop a parameter identification method to overcome this difficulty.

There are many articles [5-10] focusing on the effective methods for the estimation of coefficient $q$ within the linear elliptic equation, which can describe single-phase porous media flow with constant fluid density and viscosity

$$
-\nabla \cdot(q(x) \nabla u)=f(x) .
$$

Less work [11-14] has been done on the estimation of coefficient $q$ within the linear parabolic equation, which can describe slightly compressible single-phase flow with constant compressibility, viscosity, and porosity

$$
u_{t}-\nabla \cdot(q(x) \nabla u)=f(x, t) .
$$

It is worth noting that the coefficient $q$ is just the permeability when (4) and (5) are used to describe one-phase flow processes in porous media. For the estimation of a permeability field in the practical application, the interested readers are invited to refer to the bibliography [15-20].

Multiscale techniques have been widely investigated to reduce computation for the forward problems, such as the elliptic problems [21], the parabolic homogenization problems [22], and the Navier-Stokes equations [23], and then were extended to solve the inverse problems [24]. Wavelet multiscale method is a specific form of multiscale techniques, which has recently been used to solve various parameter estimation problems. Successful applications of this method include the Bayesian tomography $[25,26]$, the Bayesian formulations of emission tomography [27], the thermal wave tomography [28], the diffuse optical tomography [29], the velocity estimation problems of a two-dimensional wave equation [30], the permeability estimation problems of a nonlinear convection-diffusion equation [31], and the parameter estimation problems of partial differential equations [32,33]. It is shown in these papers that the performance of iterative parameter identification methods is much better with the help of the multiscale techniques.

Multigrid method, as another specific form of multiscale techniques, originally attracted interest because of its abilities to remove smooth error components and reduce the computational requirements of large numerical problems. This method works by recursively operating on the data at different grids, using the ideas of nested iterations and coarse grid correction. Multigrid method has been mainly used to solve partial differential equations, such as elliptic equation [34], modified phase field crystal equation [35], fractional diffusion equation [36], and Helmholtz equation [37]. Subsequently, researchers also applied it to a variety of imaging processing problems, for example, optical flow estimation [38], shape-from-X [39], and image matching [40].

More recently, multigrid method has been widely studied to solve parameter estimation problems [41]. In earlier work, Ranganath et al. [42] showed that the multigrid method could be applied to positron emission tomography. McCormick and Wade [43] applied a multigrid method to linearized electrical impedance tomography, and Spiliopoulos et al. [44] used a multigrid approach to estimate geometric anisotropy parameters from scattered spatial data that are obtained from environmental surveillance networks. Borcea [45] proposed a nonlinear multigrid approach for imaging the electrical conductivity and permittivity of a body, given partial, usually noisy knowledge of the Neumann-to-Dirichlet map at the boundary. Ye et al. [46] formulated the multigrid method directly in an optimization framework and used the method to solve Bayesian optical diffusion tomography. Nash [47] gave a multigrid method, which can solve a broad class of discretized optimization problems. Importantly, both the methods of Ye and Nash are based on the matching of cost functional derivatives at different grids.

In this paper, we propose a nonlinear multigrid method to estimate the permeability field within the two-phase porous media flow. This method is well suited to this permeability estimation problem for three reasons. First, the numerical examples indicate that the nonlinear multigrid method converges much faster than fixed grid methods. This is very important for the permeability estimation problem within the two-phase porous media flow because it is inherently threedimensional. Second, the optimization at each grid is done in the space-domain where positivity constraints are easily enforced, so the positivity constraints can be implemented well by multigrid method. Generally, it is important that positivity can improve estimation quality when the problem is ill-posed. In our problem, the permeability parameters to be estimated have only positive values. Finally, multigrid method can effectively overcome disturbance of local minima, and permeability estimation problem within the twophase porous media flow results in a nonconvex optimization problem; therefore, this robustness about local minima helps insure that a good solution can be reached.

A key innovation of our work is that the nonlinear multigrid method is directly formulated in an optimization framework by defining a sequence of optimization functionals at decreasing grids. In order to ensure the good convergence 
of the method, it is essential that the cost functionals at different grids be consistent. To achieve this, the coarse grid functionals are adapted by a recursive method, which guarantees that the exact fine grid solution is always a fixed point of the nonlinear multigrid method. This method greatly simplifies the application of multigrid to this permeability estimation problem and can be generalized to the other parameter estimation problems.

\section{Nonlinear Multigrid Method}

The forward problem is defined as $q \rightarrow u(x, t ; q)$; that is, a density field $u(x, t ; q)$ is derived from solving (1). We take some density values $\left\{\tilde{u}\left(x_{z}, t\right)\right\}_{z=1}^{Z}$, where $\left\{x_{z}\right\}_{z=1}^{Z}$ are some fixed points in the spatial domain, as the observation data. The permeability estimation problem is formulated as $\left\{\tilde{u}\left(x_{z}, t\right)\right\}_{z=1}^{Z} \rightarrow q$; that is, a permeability field is estimated from the limited number of observations. The parameter estimation problem is typically ill-posed, so we estimate a permeability field by minimizing the following cost functional:

$$
A(q)=\left\|u\left(x_{z}, t ; q\right)-\widetilde{u}\left(x_{z}, t\right)\right\|^{2}+\alpha\|q\|^{2},
$$

where $\alpha$ is the regularization parameter. Then, we derive a specific expression of the nonlinear multigrid method for optimization of the cost functional in (6), by starting with the two-grid case and generalizing it for the V-cycle and full multigrid cases.

2.1. Two-Grid Method. Let $q^{(0)}=q$ denote the permeability field at the finest grid, and let $q^{(k)}$ be a coarser grid representation of $q^{(0)}$ with a grid sampling period of $2^{k}$ times the finest grid sampling period. Generally, $q^{(k+1)}$ can be computed from $q^{(k)}$ by the formula

$$
q^{(k+1)}=\rrbracket_{k}^{k+1} q^{(k)}
$$

where $\square_{k}^{k+1}$ is the restriction operator.

We make the hypothesis that a cost functional $A^{(k)}\left(q^{(k)}\right)$ at grid $k$ is needed to be minimized, and there is an initial permeability field $\bar{q}^{(k)}$, which approximately minimizes this cost functional; namely,

$$
\bar{q}^{(k)} \approx \arg \min _{q^{(k)}}\left\{A^{(k)}\left(q^{(k)}\right)\right\} .
$$

We aim at computing the permeability field $\hat{q}^{(k+1)}$ at the next coarser grid and using it to improve or correct the fine grid permeability field by the following formula:

$$
\widehat{q}^{(k)} \longleftarrow \bar{q}^{(k)}+\square_{k+1}^{k}\left(\hat{q}^{(k+1)}-\rrbracket_{k}^{k+1} \bar{q}^{(k)}\right),
$$

where $\square_{k+1}^{k}$ is the prolongation operator. To compute the coarse grid permeability field $\hat{q}^{(k+1)}$, a corresponding optimization problem at the coarse grid must be formulated. To achieve it, we first choose a coarse grid cost functional $A^{(k+1)}\left(q^{(k+1)}\right)$, which approximates well to $A^{(k)}\left(q^{(k)}\right)$. Without doubt, it is very important to choose this functional, which depends on the details of the considered problem. But for the moment only assume that $A^{(k+1)}\left(q^{(k+1)}\right)$ is a reasonable approximation to the finer grid cost functional. Due to the possible discretization errors, we then solve an adjusted coarse gird optimization problem

$$
\widehat{q}^{(k+1)}=\arg \min _{q^{(k+1)}}\left\{A^{(k+1)}\left(q^{(k+1)}\right)-v^{(k+1)} q^{(k+1)}\right\},
$$

where $v^{(k+1)}$ is a row vector, which is introduced to correct the errors in the cost functional. In fact, the term $v^{(k+1)} q^{(k+1)}$ is similar to the so-called residual term in the traditional multigrid, whose function is to adjust the errors between coarse and fine grids.

The question now is how $v^{(k+1)}$ should be chosen. It is ideal that the following equality holds for all values of $q^{(k+1)}$ :

$$
\begin{aligned}
& A^{(k+1)}\left(q^{(k+1)}\right)-v^{(k+1)} q^{(k+1)}+\text { constant } \\
& \approx A^{(k)}\left(\bar{q}^{(k)}+\rrbracket_{k+1}^{k}\left(q^{(k+1)}-\rrbracket_{k}^{k+1} \bar{q}^{(k)}\right)\right),
\end{aligned}
$$

where the left side is the adjusted coarse grid cost functional, and the right side is the fine grid cost functional evaluated using the corrected result of (9). If (11) holds true, these two cost functionals will reach their minima at the same value of $q^{(k+1)}$

Generally speaking, there does not exist any row vector $v^{(k+1)}$ that makes (11) true, because the difference between the left and right sides of (11) is nonlinear. But a suitable choice of $v^{(k+1)}$ can match the derivatives of the two sides when $q^{(k+1)}=$ $\square_{\mathrm{k}}^{k+1} \bar{q}^{(k)}$. This condition yields the key expression for $v^{(k+1)}$ as follows:

$$
v^{(k+1)}=\nabla A^{(k+1)}\left(\square_{k}^{k+1} \bar{q}^{(k)}\right)-\nabla A^{(k)}\left(\bar{q}^{(k)}\right) \rrbracket_{k+1}^{k},
$$

where $\nabla A^{(k)}\left(q^{(k)}\right), \nabla A^{(k+1)}\left(q^{(k+1)}\right)$ are, respectively, the gradient vectors of $A^{(k)}\left(q^{(k)}\right), A^{(k+1)}\left(q^{(k+1)}\right)$. This condition is essential to assure that the optimum solution of (10) is a fixed point of this two-grid method and is illustrated graphically in Figure 1. It is worth noting that in (12) the restriction operator $\square_{k}^{k+1}$, which comes from the chain rule of differentiation, actually plays the role of a prolongation operator because it multiplies the gradient vector on the right. Most importantly, (12) holds for any choice of restriction and prolongation operators.

2.2. Multigrid Method. Multigrid method can be implemented by recursively applying the two-grid method, and two recursions known as $\mathrm{V}$-cycle and full multigrids are used in this section. Both these two multigrid methods move back and forth through coarse and fine grid in characteristic patterns as shown in Figure 2.

The V-cycle multigrid method is a straightforward generalization of the two-grid method. In fact, the coarse gird optimization problem in the two-grid method is recursively solved by another two-grid method; then the V-cycle multigrid method can be got, as shown in Figure 2(a). 


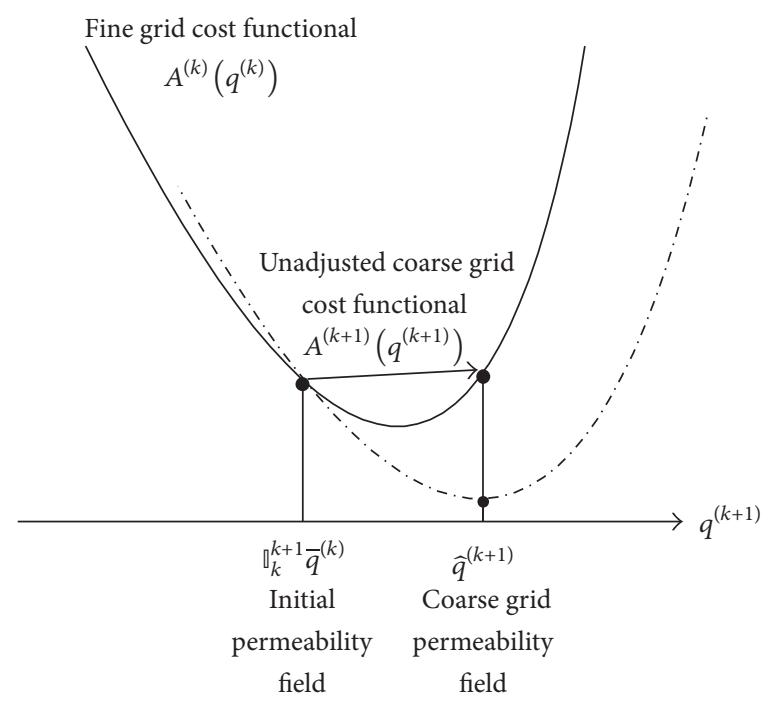

(a) The derivatives of the two sides in (11) are different

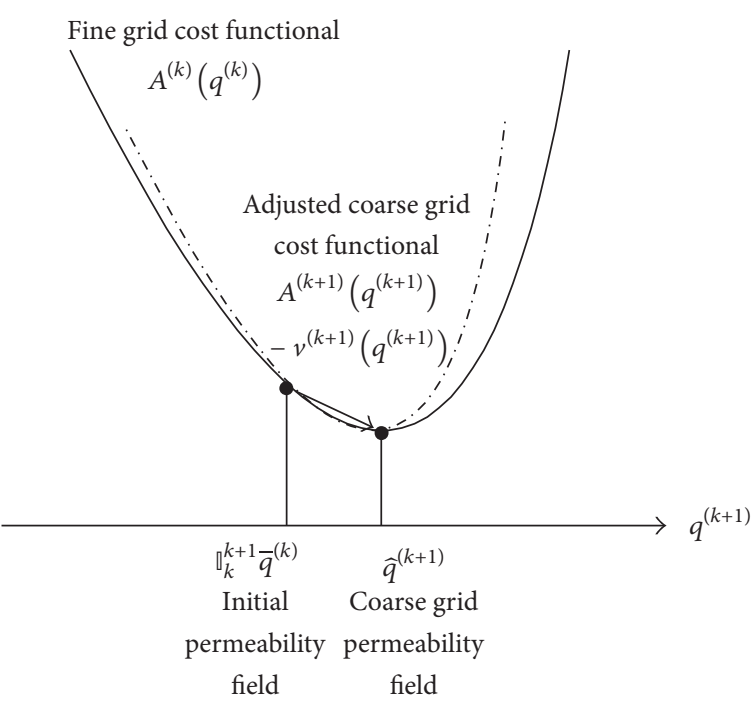

(b) The derivatives of the two sides in (11) are equal

Figure 1: The function of the term $v^{(k+1)} q^{(k+1)}$.

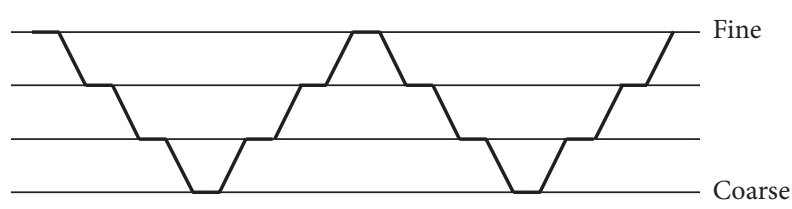

(a) V-cycle multigrid

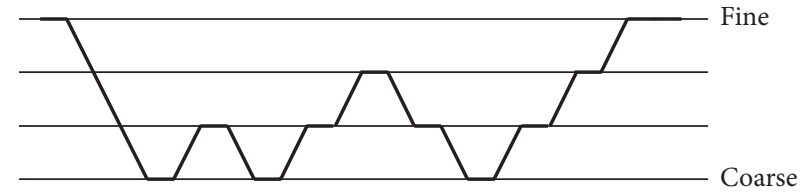

(b) Full multigrid

FIGURE 2: The description of the multigrid methods.

The full multigrid method, as shown in Figure 2(b), is on the basis of recursive calls of both the $\mathrm{V}$-cycle and full multigrids. This structure causes this method to initially move to the coarsest grid. We prolongate the obtained coarsest grid permeability field to the second coarsest grid and use it as the initial permeability field for the second coarsest grid optimization problem, which is then solved by a V-cycle multigrid. Repeat this process until the final permeability field is got at the finest grid.

\section{Simulation Results}

In this section, we report simulation results on four numerical examples in two dimensions. We perform the simulations on a $2.6 \mathrm{GHz}$ PC with $4 \mathrm{~GB}$ RAM in MATLAB 2012b environment under Windows 7. In our simulations, the permeability field $q$ is estimated both with the nonlinear multigrid method (NMGM) and with the fixed grid method (FGM, i.e., the Levenberg-Marquardt method [31]). For the estimation, we use the parameters as follows:

$$
\begin{aligned}
i(x) & =\sin \left(\pi x_{1}\right) \sin \left(\pi x_{2}\right), \\
b(x, t) & =0, \\
f(x, t) & =0, \\
\Omega & =[0,1] \times[0,1] .
\end{aligned}
$$

In the first two examples, the spatial and temporal step sizes $\Delta x_{1}, \Delta x_{2}$, and $\Delta t$ are, respectively, chosen to be $1 / 8,1 / 8$, and $0.0025 \mathrm{~s}$, and the initial permeability field $q^{0}$ is chosen to be constant 2. The artificial Gaussian noise with level $1 \%$ is added to the measurement data. In the last two examples. The spatial and temporal step sizes $\Delta x_{1}, \Delta x_{2}$, and $\Delta t$ are, respectively, chosen to be $1 / 24,1 / 24$, and $0.005 \mathrm{~s}$, and the initial permeability field $q^{0}$ is chosen to be constant 5 . The level of Gaussian noise added to the measurement data is chosen to be $2 \%$. For the nonlinear multigrid method, the regularization parameters in the coarsest and finest grids are, respectively, chosen as $\alpha=10^{-4}$ and $\alpha=10^{-8}$. For the fixed grid method, the regularization parameter is chosen as $\alpha=10^{-4}$. This is because the initial permeability field is not very good; a regularization parameter value smaller than $10^{-4}$ will lead to the divergence of the fixed grid method.

Example 1. In the first example, the nonlinear function $N(u)=u^{2}+u+1$, and the exact permeability field to be estimated, $q(x)$, is piecewise constant

$$
q(x)= \begin{cases}1, & x \in[0,0.5] \times[0,0.5], \\ 2, & x \in[0,0.5] \times[0.5,1], \\ 3, & x \in[0.5,1] \times[0,0.5], \\ 4, & x \in[0.5,1] \times[0.5,1] .\end{cases}
$$




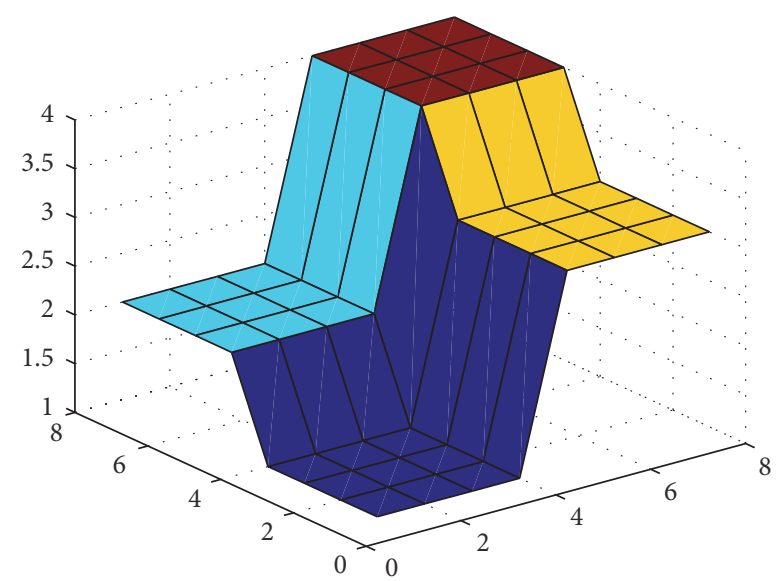

(a) Exact permeability field

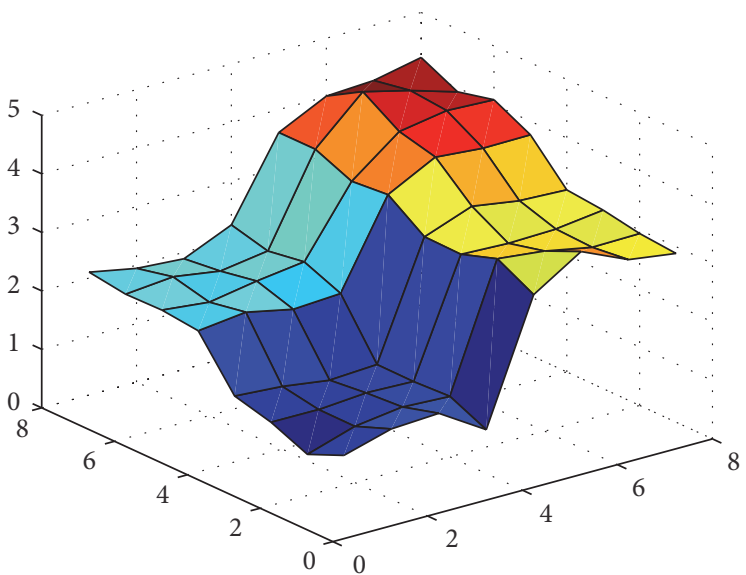

(b) Estimated permeability field using NMGM

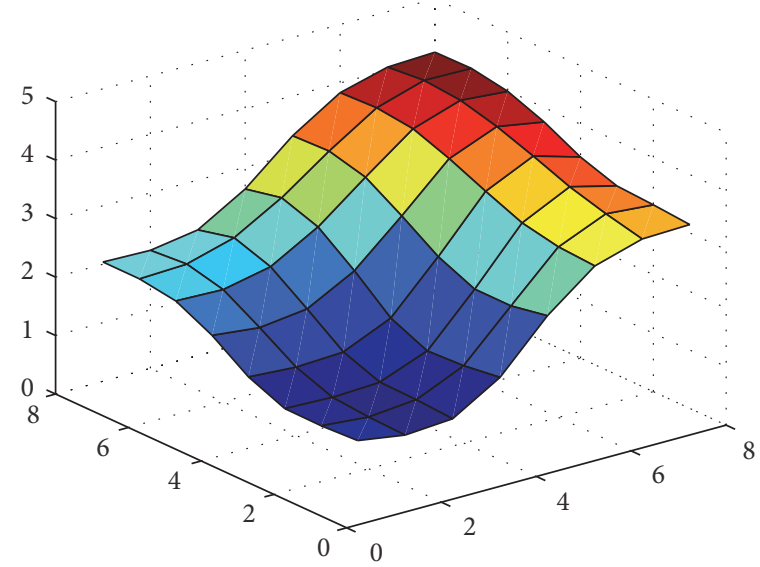

(c) Estimated permeability field using FGM

FIGURE 3: The exact permeability field and estimated permeability fields in Example 1.

TABLE 1: Comparison of NMGM and FGM in the small-scale models.

\begin{tabular}{lccc}
\hline $\begin{array}{l}\text { Example } \\
\text { number }\end{array}$ & $\begin{array}{c}\text { Estimation } \\
\text { method }\end{array}$ & Relative error & CPU time (s) \\
\hline Example 1 & NMGM & 0.0421 & 4.975 \\
& FGM & 0.1253 & 13.079 \\
Example 2 & NMGM & 0.0634 & 4.815 \\
& FGM & 0.2725 & 9.199 \\
\hline
\end{tabular}

Figure 3 displays the exact permeability field, the estimated permeability field using the nonlinear multigrid method, and the estimated permeability field using the fixed grid method. To validate the merits of the nonlinear multigrid method, the relative errors and CPU times in this example are listed in Table 1.

By observing Table 1, it is clear that the nonlinear multigrid method is much faster than the fixed grid method. Furthermore, by comparing Figures 3(b) and 3(c), it appears that the estimated permeability field using the nonlinear multigrid method is much better than the one using the fixed grid method. Thus, we conclude that the nonlinear multigrid method is efficient, accurate, and stable.

Example 2. In this example, the nonlinear function $N(\nabla u)=$ $1+0.1|\nabla u|^{2}$. In oil reservoirs the permeability field usually has greatly large jumps, so the exact permeability field $q(x)$ is piecewise constant

$$
q(x)= \begin{cases}1, & x \in[0,0.5] \times[0,0.5], \\ 2, & x \in[0,0.5] \times[0.5,1], \\ 5, & x \in[0.5,1] \times[0,0.5], \\ 10, & x \in[0.5,1] \times[0.5,1] .\end{cases}
$$

Figure 4 shows the exact permeability field, the estimated permeability field using the nonlinear multigrid method, and the estimated permeability field using the fixed grid method, and Table 1 also lists the relative errors and CPU times in this example.

It should be clear from this example that even if $N$ is highly nonlinear, the nonlinear multigrid method remains efficient, accurate, and stable. In fact, when the permeability 


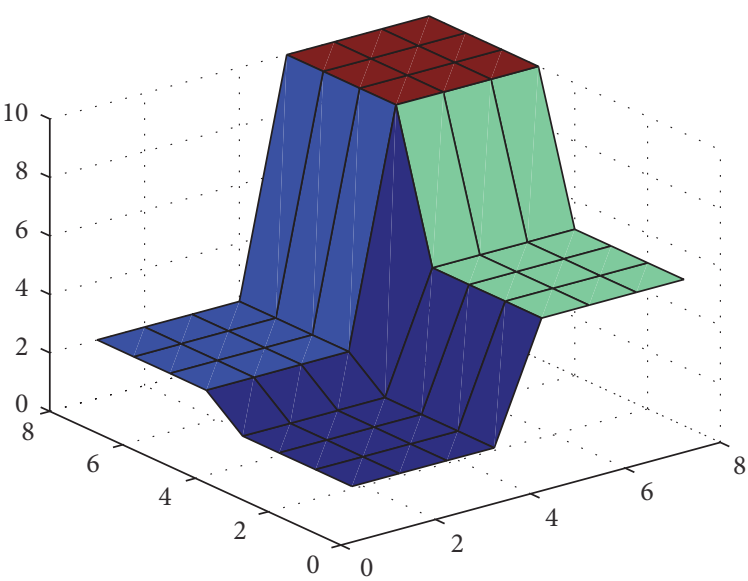

(a) Exact permeability field

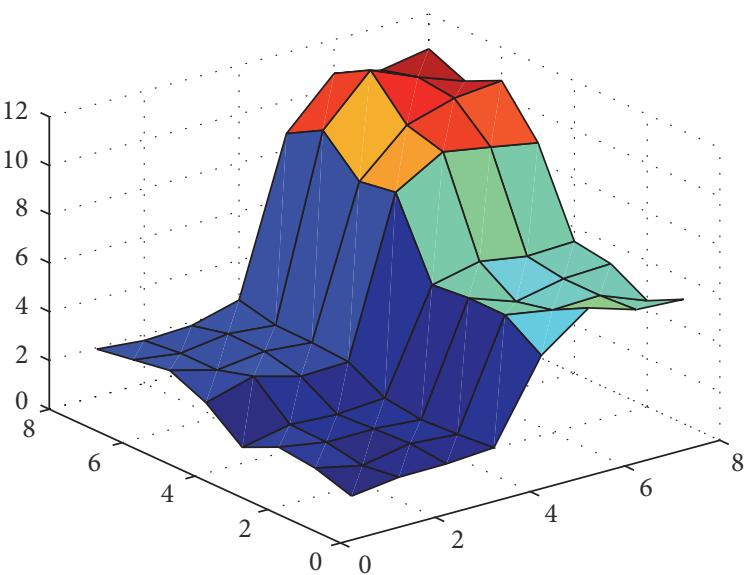

(b) Estimated permeability field using NMGM

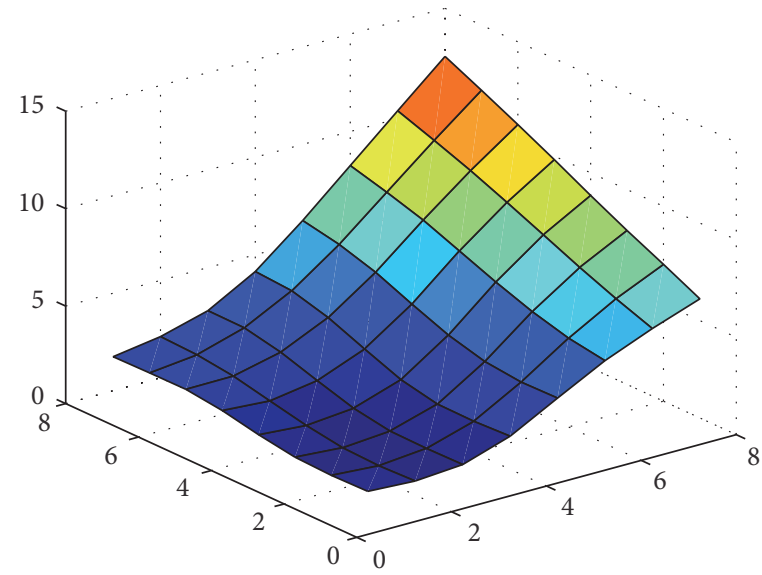

(c) Estimated permeability field using FGM

FIGURE 4: The exact permeability field and estimated permeability fields in Example 2.

field has greatly large jumps, the permeability field estimated with the fixed grid method is not satisfactory. This may be seen by comparing Figure 4(c), which presents an oversmooth permeability field, with Figure 4(b), which presents an acceptable permeability field. Therefore, we conclude that the multigrid procedure is effective and even necessary in oil reservoir simulation.

Example 3. In this example, the nonlinear function $N(u)=$ $u^{4}-u^{3}+u^{2}-u+1$. The exact permeability field to be estimated, as shown in Figure 5(a), is a vertical stratified medium containing two interfaces, where the permeabilities from left to right are, respectively, 3.91, 5.03, and 6.18. The estimated permeability field using the nonlinear multigrid method is shown in Figure 5(b), and the relative errors and CPU times in this example are listed in Table 2.

Example 4. In this example, the nonlinear function $N(\nabla u)=$ $1 /\left(1-0.1|\nabla u|^{2}\right)$. The exact permeability field to be estimated, as shown in Figure 6(a), is the model of two anomalous bodies in a homogeneous medium with a permeability of 6.27 . The anomalous bodies have the permeabilities of 3.56 and 4.79 , respectively. Figure 6(b) shows the estimated permeability
TABLE 2: Comparison of NMGM and FGM in the large-scale models.

\begin{tabular}{lccc}
\hline $\begin{array}{l}\text { Example } \\
\text { number }\end{array}$ & $\begin{array}{c}\text { Estimation } \\
\text { method }\end{array}$ & Relative error & CPU time (s) \\
\hline Example 3 & NMGM & 0.0316 & 367.354 \\
& FGM & 0.1183 & 571.284 \\
Example 4 & NMGM & 0.0285 & 291.901 \\
& FGM & 0.1077 & 503.251 \\
\hline
\end{tabular}

field using the nonlinear multigrid method, and Table 2 also lists the relative errors and CPU times in this example.

It is evident from these two examples and the ones previously discussed that the merits of the nonlinear multigrid method, such as the computational efficiency, computational accuracy, and antinoise ability, still exist for the highresolution and complicated permeability fields.

\section{Discussion}

Estimation of the permeability field within the porous media flow is not only a very important research topic itself but also 


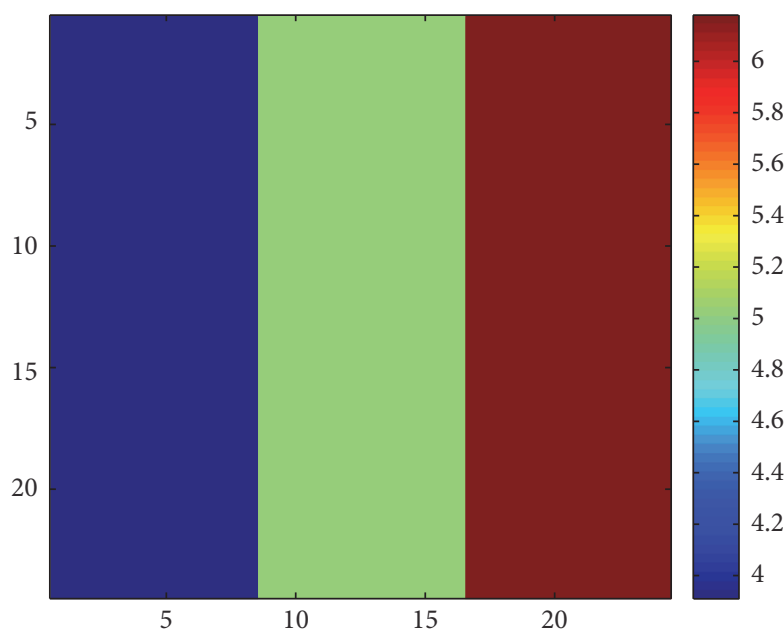

(a) Exact permeability field

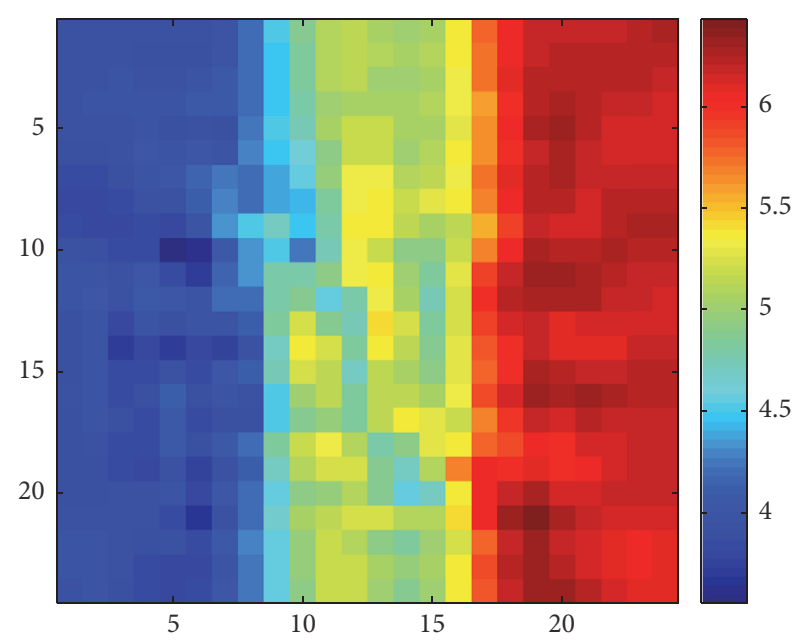

(b) Estimated permeability field using NMGM

FIgURE 5: The exact permeability field and estimated permeability field in Example 3.

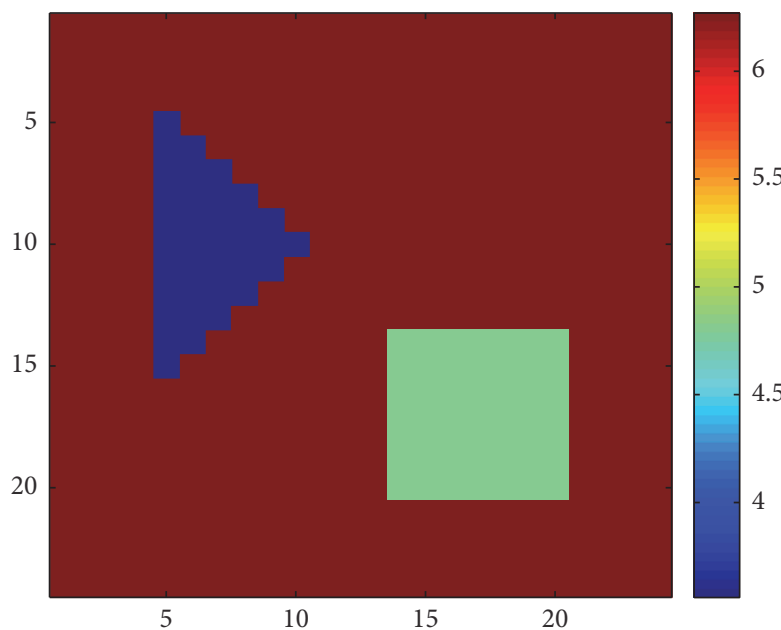

(a) Exact permeability field

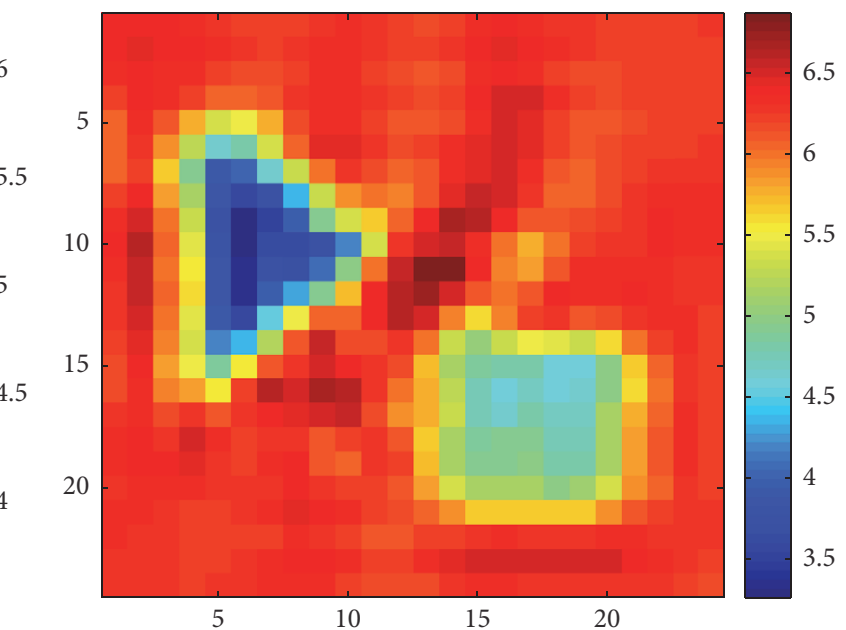

(b) Estimated permeability field using NMGM

FIgURE 6: The exact permeability field and estimated permeability field in Example 4.

has applications in a variety of domains, such as reservoir simulation and oil and gas exploration. Various methods had been presented to solve the problem of estimating the permeability field within the one-phase porous media flow. Within the growing body of work concerning multiphase porous media flow, few studies have focused on the issue of estimating the permeability field within the two-phase porous media flow. This paper has proposed a new method based on multigrid to estimate the permeability field for the nonlinear diffusion equation within the two-phase porous media flow. In this nonlinear multigrid method, the cost functionals at different grids are dynamically adjusted to be compatible, so that the exact fine grid solution can be always a fixed point of the nonlinear multigrid method. In comparison with the fixed grid method, the main advantages of this approach are that it can dramatically reduce the computational complexity and improve the estimation quality. Moreover, by taking into consideration observation noise, the robustness of the method has been demonstrated. This approach works entirely in an optimization framework; therefore, it provides a sensible way to estimate the permeability field within the twophase porous media flow, which has a clear potential applicability to a wide variety of parameter estimation problems.

\section{Competing Interests}

The authors declare that they have no competing interests.

\section{Acknowledgments}

This work was supported by the National Natural Science Foundation of China (11271102), the Science and Technology Funds for the Universities in Hebei Province of China (Z2015039), the Fundamental Research Funds for the Central 
Universities (N142303011), the PhD Foundation of Northeast University at Qinhuangdao (XNB2015002).

\section{References}

[1] M. Cuypers, O. Dubrule, P. Lamy, and R. Bissell, "Optimal choice of inversion parameters for history-matching with the pilot method," in Proceedings of the 6th European Conference on the Mathematics of Oil Recovery, Peebles, UK, September 1998.

[2] A. A. Grimstad, T. Mannseth, J. E. Nordtvedt, and G. Nævdal, "Reservoir characterization through scale splitting," in Proceedings of the 7th European Conference on the Mathematics of Oil Recovery (ECMOR '00), 2000.

[3] A. C. Reynolds, N. He, L. Chu, and D. S. Oliver, "Reparameterization techniques for generating reservoir descriptions conditioned to variograms and well-test pressure data," SPE Journal, vol. 1, no. 4, pp. 413-426, 1996.

[4] P. C. Shah, G. R. Gavalas, and J. H. Seinfeld, "Error analysis in history matching: the optimum level of parameterization," Society of Petroleum Engineers Journal, vol. 18, no. 3, pp. 219228, 1978.

[5] R. Luce and S. Perez, "Parameter identification for an elliptic partial differential equation with distributed noisy data," Inverse Problems, vol. 15, no. 1, pp. 291-307, 1999.

[6] K. Ito and K. Kunisch, "The augmented Lagrangian method for parameter estimation in elliptic systems," SIAM Journal on Control and Optimization, vol. 28, no. 1, pp. 113-136, 1990.

[7] R. Acar, "Identification of the coefficient in elliptic equations," SIAM Journal on Control and Optimization, vol. 31, no. 5, pp. 1221-1244, 1993.

[8] C. Chicone and J. Gerlach, "A note on the identifiability of distributed parameters in elliptic equations," SIAM Journal on Mathematical Analysis, vol. 18, no. 5, pp. 1378-1384, 1987.

[9] R. Rannacher and B. Vexler, "A priori error estimates for the finite element discretization of elliptic parameter identification problems with pointwise measurements," SIAM Journal on Control and Optimization, vol. 44, no. 5, pp. 1844-1863, 2005.

[10] T. F. Chan and X.-C. Tai, "Identification of discontinuous coefficients in elliptic problems using total variation regularization," SIAM Journal on Scientific Computing, vol. 25, no. 3, pp. 881904, 2003.

[11] A. Binder, H. W. Engl, C. W. Groetsch, A. Neubauer, and O. Scherzer, "Weakly closed nonlinear operators and parameter identification in parabolic equations by Tikhonov regularization," Applicable Analysis, vol. 55, no. 3-4, pp. 215-234, 1994.

[12] Y. L. Keung and J. Zou, "Numerical identifications of parameters in parabolic systems," Inverse Problems, vol. 14, no. 1, pp. 83-100, 1998.

[13] S. Kitamura and S. Nakagiri, "Identifiability of spatially-varying and constant parameters in distributed systems of parabolic type," SIAM Journal on Control and Optimization, vol. 15, no. 5, pp. 785-802, 1977.

[14] J. R. Cannon and D. Zachmann, "Parameter determination in parabolic partial differential equations from overspecified boundary data," International Journal of Engineering Science, vol. 20, no. 6, pp. 779-788, 1982.

[15] R. I. Hume, R. Dingledine, and S. F. Heinemann, "Identification of a site in glutamate receptor subunits that controls calcium permeability," Science, vol. 253, no. 5023, pp. 1028-1031, 1991.

[16] P. Yu, B. Liu, and T. Kodadek, "A convenient, high-throughput assay for measuring the relative cell permeability of synthetic compounds," Nature Protocols, vol. 2, no. 1, pp. 23-30, 2007.
[17] S. P. Cramer and H. B. W. Larsson, "Accurate determination of blood-brain barrier permeability using dynamic contrastenhanced T1-weighted MRI: a simulation and in vivo study on healthy subjects and multiple sclerosis patients," Journal of Cerebral Blood Flow and Metabolism, vol. 34, no. 10, pp. 16551665, 2014.

[18] D. H. Jo, R. Lee, J. H. Kim, H. O. Jun, T. G. Lee, and J. H. Kim, "Real-time estimation of paracellular permeability of cerebral endothelial cells by capacitance sensor array," Scientific Reports, vol. 5, Article ID 11014, 2015.

[19] M. Saemi, M. Ahmadi, and A. Y. Varjani, "Design of neural networks using genetic algorithm for the permeability estimation of the reservoir," Journal of Petroleum Science and Engineering, vol. 59, no. 1-2, pp. 97-105, 2007.

[20] F. Ingels, B. Beck, M. Oth, and P. Augustijns, "Effect of simulated intestinal fluid on drug permeability estimation across Caco2 monolayers," International Journal of Pharmaceutics, vol. 274, no. 1-2, pp. 221-232, 2004.

[21] T. Y. Hou and X.-H. Wu, "A multiscale finite element method for elliptic problems in composite materials and porous media," Journal of Computational Physics, vol. 134, no. 1, pp. 169-189, 1997.

[22] P. Ming and P. Zhang, "Analysis of the heterogeneous multiscale method for parabolic homogenization problems," Mathematics of Computation, vol. 76, no. 257, pp. 153-177, 2007.

[23] V. John and S. Kaya, "A finite element variational multiscale method for the Navier-Stokes equations," SIAM Journal on Scientific Computing, vol. 26, no. 5, pp. 1485-1503, 2005.

[24] M. AlQuraishi, G. Koytiger, A. Jenney, G. MacBeath, and P. K. Sorger, "A multiscale statistical mechanical framework integrates biophysical and genomic data to assemble cancer networks," Nature Genetics, vol. 46, no. 12, pp. 1363-1371, 2014.

[25] M. Bhatia, W. C. Karl, and A. S. Willsky, "A wavelet-based method for multiscale tomographie reconstruction," IEEE Transactions on Medical Imaging, vol. 15, no. 1, pp. 92-101, 1996.

[26] M. Bhatia, W. C. Karl, and A. S. Willsky, “Tomographic reconstruction and estimation based on multiscale natural-pixel bases," IEEE Transactions on Image Processing, vol. 6, no. 3, pp. 463-478, 1997.

[27] T. Frese, C. A. Bouman, and K. Sauer, "Adaptive wavelet graph model for Bayesian tomographic reconstruction," IEEE Transactions on Image Processing, vol. 11, no. 7, pp. 756-770, 2002.

[28] E. L. Miller, L. Nicolaides, and A. Mandelis, "Nonlinear inverse scattering methods for thermal-wave slice tomography: a wavelet domain approach," Journal of the Optical Society of America A, vol. 15, no. 6, pp. 1545-1556, 1998.

[29] F. Dubot, Y. Favennec, B. Rousseau, and D. R. Rousse, "A wavelet multi-scale method for the inverse problem of diffuse optical tomography," Journal of Computational and Applied Mathematics, vol. 289, pp. 267-281, 2015.

[30] H. S. Fu and B. Han, "A wavelet multiscale method for the inverse problems of a two-dimensional wave equation," Inverse Problems in Science and Engineering, vol. 12, no. 6, pp. 643-656, 2004.

[31] T. Liu, "Reconstruction of a permeability field with the wavelet multiscale-homotopy method for a nonlinear convectiondiffusion equation," Applied Mathematics and Computation, vol. 275, pp. 432-437, 2016.

[32] H. Fu, B. Han, and H. Liu, "A wavelet multiscale iterative regularization method for the parameter estimation problems of partial differential equations," Neurocomputing, vol. 104, pp. 138-145, 2013. 
[33] T. Liu, "A wavelet multiscale-homotopy method for the parameter identification problem of partial differential equations," Computers and Mathematics with Applications, vol. 71, no. 7, pp. 1519-1523, 2016.

[34] S. R. Fulton, P. E. Ciesielski, and W. H. Schubert, "Multigrid methods for elliptic problems: a review," Monthly Weather Review, vol. 114, no. 5, pp. 943-959, 1986.

[35] A. Baskaran, Z. Hu, J. S. Lowengrub, C. Wang, S. M. Wise, and P. Zhou, "Energy stable and efficient finite-difference nonlinear multigrid schemes for the modified phase field crystal equation," Journal of Computational Physics, vol. 250, pp. 270292, 2013

[36] H.-K. Pang and H.-W. Sun, "Multigrid method for fractional diffusion equations," Journal of Computational Physics, vol. 231, no. 2, pp. 693-703, 2012.

[37] C. C. Stolk, M. Ahmed, and S. K. Bhowmik, "A multigrid method for the Helmholtz equation with optimized coarse grid corrections," SIAM Journal on Scientific Computing, vol. 36, no. 6, pp. A2819-A2841, 2014.

[38] P. Hellier, C. Barillot, E. Mémin, and P. Pérez, "Hierarchical estimation of a dense deformation field for 3-D robust registration," IEEE Transactions on Medical Imaging, vol. 20, no. 5, pp. 388402, 2001.

[39] R. Kimmel and I. Yavneh, "An algebraic multigrid approach for image analysis," SIAM Journal on Scientific Computing, vol. 24, no. 4, pp. 1218-1231, 2003.

[40] S. Henn and K. Witsch, "A multigrid approach for minimizing a nonlinear functional for digital image matching," Computing, vol. 64, no. 4, pp. 339-348, 2000.

[41] U. Ascher and E. Haber, "A multigrid method for distributed parameter estimation problems," Electronic Transactions on Numerical Analysis, vol. 18, pp. 1-18, 2003.

[42] M. V. Ranganath, A. P. Dhawan, and N. Mullani, "A multigrid expectation maximization reconstruction algorithm for positron emission tomography," IEEE Transactions on Medical Imaging, vol. 7, no. 4, pp. 273-278, 1988.

[43] S. F. McCormick and J. G. Wade, "Multigrid solution of a linearized, regularized least-squares problem in electrical impedance tomography," Inverse Problems, vol. 9, no. 6, pp. 697713, 1993.

[44] I. Spiliopoulos, D. T. Hristopulos, M. P. Petrakis, and A. Chorti, "A multigrid method for the estimation of geometric anisotropy in environmental data from sensor networks," Computers and Geosciences, vol. 37, no. 3, pp. 320-330, 2011.

[45] L. Borcea, "A nonlinear multigrid for imaging electrical conductivity and permittivity at low frequency," Inverse Problems, vol. 17, no. 2, pp. 329-359, 2001.

[46] J. C. Ye, C. A. Bouman, K. J. Webb, and R. P. Millane, "Nonlinear multigrid algorithms for Bayesian optical diffusion tomography," IEEE Transactions on Image Processing, vol. 10, no. 6, pp. 909-922, 2001.

[47] S. G. Nash, "A multigrid approach to discretized optimization problems," Optimization Methods and Software, vol. 14, no. 1-2, pp. 99-116, 2000. 


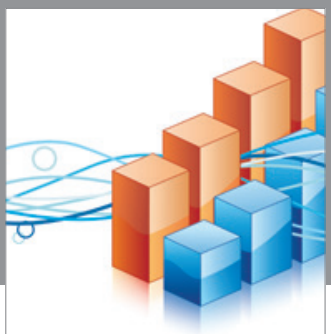

Advances in

Operations Research

vatem alat4

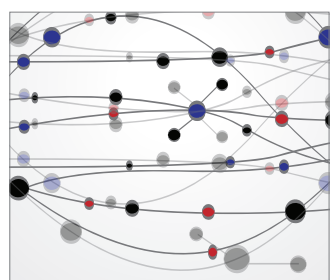

\section{The Scientific} World Journal
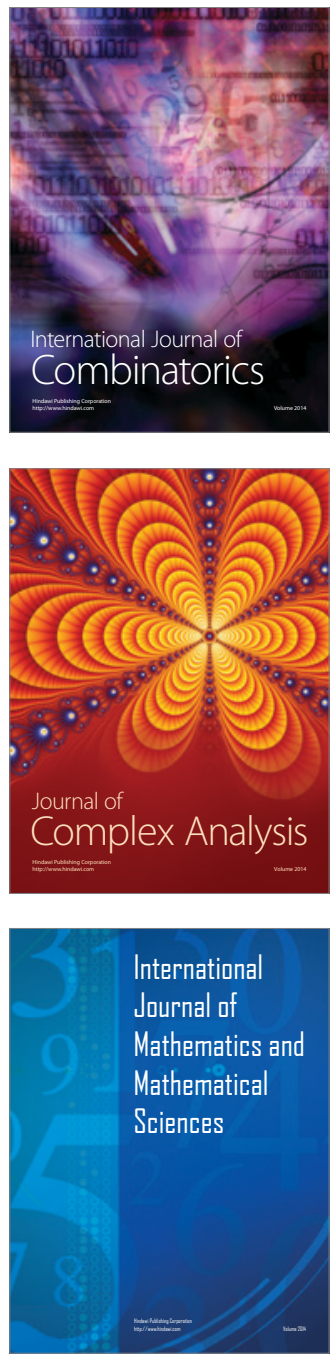
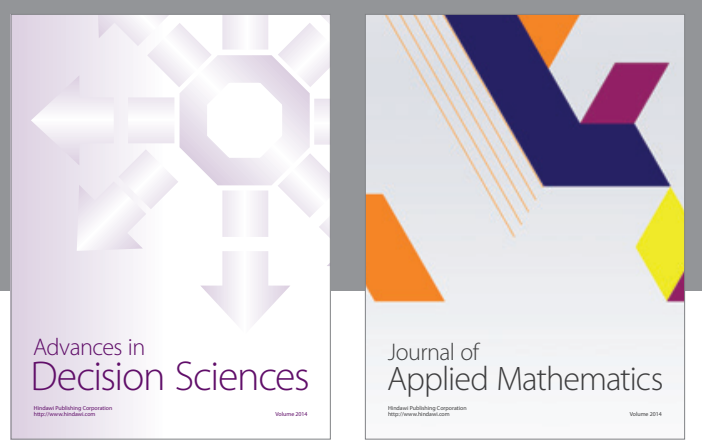

Algebra

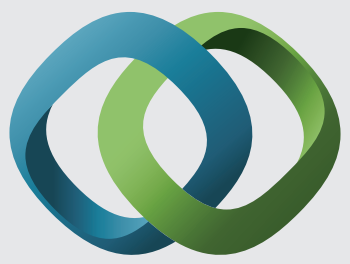

\section{Hindawi}

Submit your manuscripts at

https://www.hindawi.com
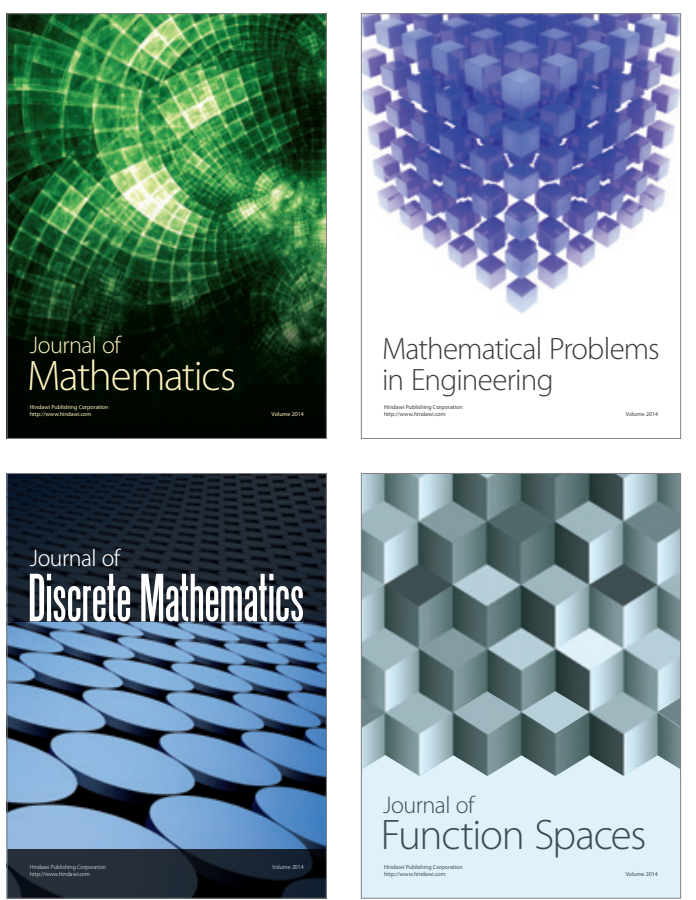

Mathematical Problems in Engineering
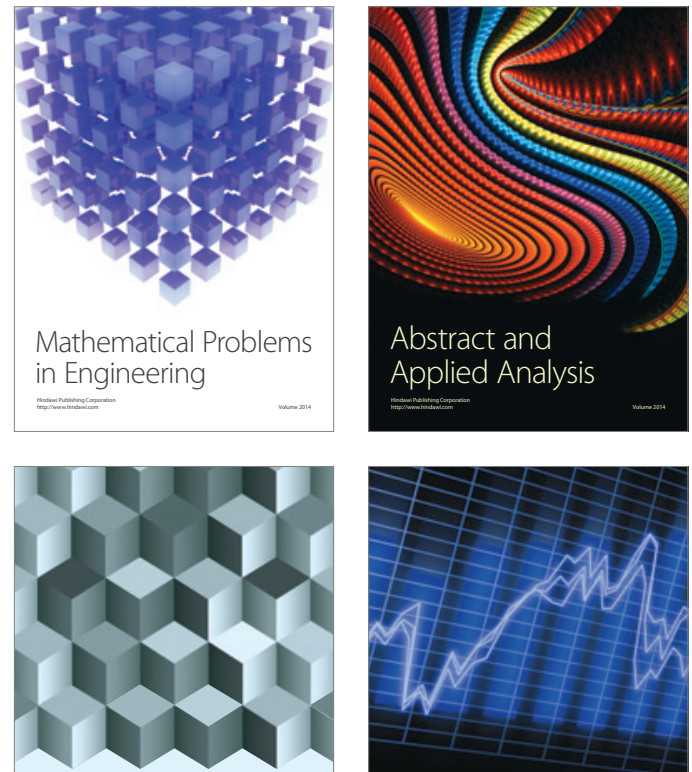

Journal of

Function Spaces

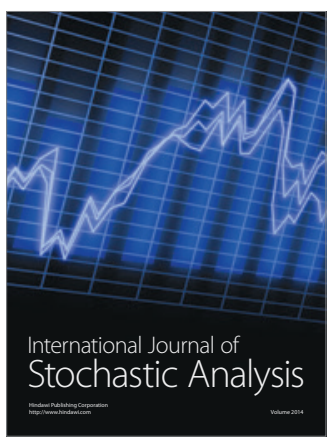

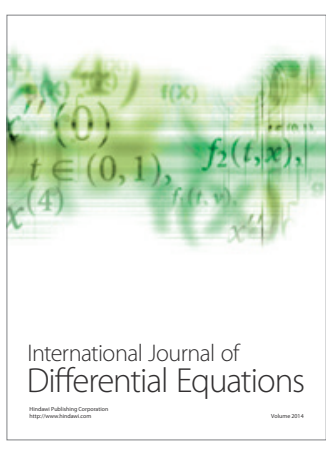
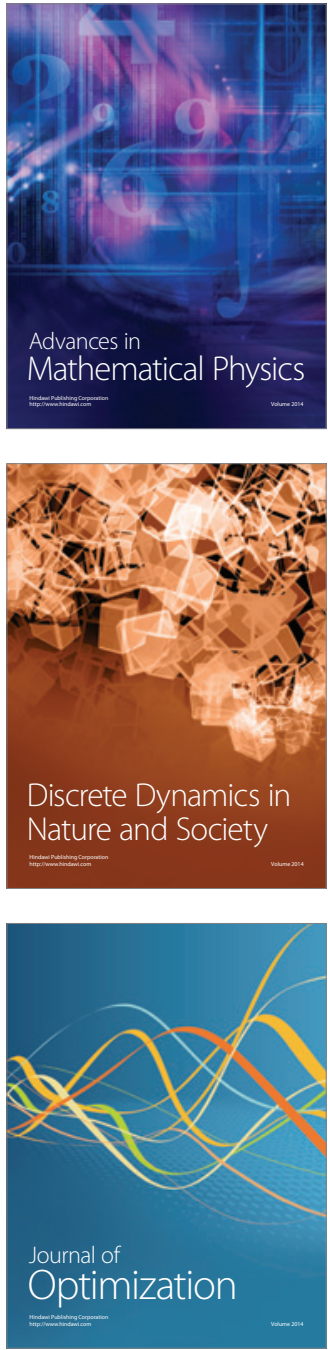